TRANSACTIONS OF THE

AMERICAN MATHEMATICAL SOCIETY

Volume 354, Number 2, Pages 613-630

S 0002-9947(01)02884-7

Article electronically published on September 28, 2001

\title{
SUFFICIENT CONDITIONS FOR ZERO-ONE LAWS
}

\author{
JASON P. BELL
}

\begin{abstract}
We generalize a result of Bateman and Erdős concerning partitions, thereby answering a question of Compton. From this result it follows that if $\mathcal{K}$ is a class of finite relational structures that is closed under the formation of disjoint unions and the extraction of components, and if it has the property that the number of indecomposables of size $n$ is bounded above by a polynomial in $n$, then $\mathcal{K}$ has a monadic second order 0-1 law. Moreover, we show that if a class of finite structures with the unique factorization property is closed under the formation of direct products and the extraction of indecomposable factors, and if it has the property that the number of indecomposables of size at most $n$ is bounded above by a polynomial in $\log n$, then this class has a first order 0-1 law. These results cover all known natural examples of classes of structures that have been proved to have a logical 0-1 law by Compton's method of analyzing generating functions.
\end{abstract}

\section{INTRODUCTION}

This paper is concerned with finding conditions on classes $\mathcal{K}$ of finite structures 1 which guarantee that the probability of a property holding in $\mathcal{K}$ will always be either 0 or 1 , provided the property is expressible in a suitable logic.

The definition of the probability of a property holding involves counting the number of structures of size $n$ in $\mathcal{K}$, as well as in the subclass defined by the property. In this paper counting is always done up to isomorphism.

1.1. Additive results. Compton noted in the 1980s that classes $\mathcal{K}$ of finite relational structures studied in combinatorics are frequently closed under the formation of disjoint unions and the extraction of components ${ }^{2}$ for example, consider the class of finite acyclic graphs, the class of finite binary forests, etc 3 Such a class $\mathcal{K}$ is said to be additively adequate 4 and satisfies the following fundamental identity.

Received by the editors April 10, 2000 and, in revised form, May 18, 2001.

1991 Mathematics Subject Classification. Primary 60F20; Secondary 05A16.

I am indebted to Stan Burris for pointing out that results obtained in the additive case lift to the multiplicative case, to John Lawrence for helping with an application, and to the referee for valuable comments regarding the presentation.

${ }^{1} \mathrm{~A}$ structure will mean a structure for a first-order language, that is, a set equipped with appropriate relations, functions, and constants.

${ }^{2}$ Compton defined the notion of a connected relational structure, and noted that every relational structure is uniquely a disjoint union of components, where a component is a maximal connected substructure.

${ }^{3}$ The fact that the size of the disjoint union of two structures is the sum of their sizes leads to the classification of counting problems for such classes as belonging to the additive case.

${ }^{4} \mathrm{~A}$ more general definition of adequate appears in 2. The important natural examples are covered by our definition, and all the theorems in this paper hold in the more general case. 


\section{Identity 1.}

$$
\sum_{n=0}^{\infty} f_{\mathcal{K}}(n) x^{n}=\prod_{n=1}^{\infty}\left(1-x^{n}\right)^{-g_{\mathcal{K}}(n)},
$$

where $f_{\mathcal{K}}(0)=1$, and otherwise $f_{\mathcal{K}}(n)$ counts the number of structures of size $n$ in $\mathcal{K}$, and where $g_{\mathcal{K}}(n)$ counts the number of components of size $n$ in $\mathcal{K}$. Identity 1 plays a crucial role in Compton's work.

Compton [6] proved the following.

Theorem 1.1. If $\mathcal{K}$ is an additively adequate class of finite relational structures and

$$
f_{\mathcal{K}}(n-1) / f_{\mathcal{K}}(n) \rightarrow 1,
$$

then $\mathcal{K}$ has a monadic second order 0-1 law, that is, given any monadic second order sentence, the probability that it holds in $\mathcal{K}$ is always either 0 or 15

Using Theorem 1.1 and a result of Bateman and Erdős (see the remark following Theorem 1.3 below), he derived the following.

Theorem 1.2. If $\mathcal{K}$ is additively adequate and $g_{\mathcal{K}}(n) \leq 1$ for all $n$, then $\mathcal{K}$ has a monadic second order 0-1 law.

By a slight modification of the Bateman and Erdős proof one can weaken the hypothesis of Theorem 1.2 to $g_{\mathcal{K}}(n)=\mathrm{O}(1)$. However Compton suspected that much more might be true and asked if one could weaken the hypothesis to ' $g_{\mathcal{K}}(n)=$ $\mathrm{O}\left(n^{k}\right)$ for some $k$ '-see Question 8.3 of [5].

To answer this in the affirmative we prove the following generalization of the Bateman and Erdős result (this theorem belongs to additive number theory).

Theorem 1.3. Suppose $f$ and $g$ are functions with nonnegative integer values satisfying Identity 1, and $\operatorname{gcd}(\{n: g(n) \neq 0\})=1$. If $g(n)=\mathrm{O}\left(n^{k}\right)$ for some $k$, then $f(n-1) / f(n) \rightarrow 1$.

Remark 1.4. Bateman and Erdös proved this under the assumption $g(n) \leq 1$ for all $n$ - see section 4.2 of [2].

Combining this with Theorem 1.1 leads to our first result on 0-1 laws.

Theorem 1.5. If $\mathcal{K}$ is an additively adequate class of finite relational structures such that $g_{\mathcal{K}}(n)=\mathrm{O}\left(n^{k}\right)$ for some $k$, then $\mathcal{K}$ has a monadic second order 0-1 law.

1.2. Multiplicative results. It was later observed by Compton that there were important parallels between many of the classes of structures studied in combinatorics and the classes studied in algebra. One often has that classes $\mathcal{K}$ studied in algebra are closed under the formation of direct products and the extraction of directly indecomposable factors, and they have the unique factorization property 6

\footnotetext{
${ }^{5}$ In the additive case, the probability that a sentence holds is defined to be the limit as $n \rightarrow \infty$ of the proportion of structures of size $n$ in $\mathcal{K}$ that satisfy the sentence, provided such limit exists. (Only those $n$ for which $\mathcal{K}$ has structures of size $n$ are considered when determining if the sequence of proportions has a limit.)

${ }^{6}$ The determination of whether or not a class $\mathcal{K}$ of finite structures is such that every member has the unique factorization property has been a subject of much interest, both in the case of algebraic structures (i.e., no relations) by Kurosh, Ore, Tarski, Jónsson, McKenzie, etc., and in the case of purely relational structures by Lovász, McKenzie, etc.
} 
for example, finite Abelian groups, finite Boolean algebras, finite semisimple algebras, etc 7 Such a class $\mathcal{K}$ is said to be multiplicatively adequate and satisfies the following fundamental identity (see Definition 8.8 of [2]).

\section{Identity 2.}

$$
\sum_{n=1}^{\infty} f_{\mathcal{K}}(n) n^{-s}=\prod_{n=2}^{\infty}\left(1-n^{-s}\right)^{-g_{\mathcal{K}}(n)}=\exp \left(\sum_{n=2}^{\infty} h_{\mathcal{K}}(n) n^{-s}\right),
$$

where $f_{\mathcal{K}}(1)=1$, and otherwise $f_{\mathcal{K}}(n)$ counts the number of structures of size $n$ in $\mathcal{K} ; g_{\mathcal{K}}(n)$ counts the number of indecomposable structures of size $n$ in $\mathcal{K}$; and

$$
h_{\mathcal{K}}(n)=\sum_{j^{\ell}=n} g_{\mathcal{K}}(j) / \ell \quad \text { for } n \geq 2 .
$$

Burris, Compton, Odlyzko, and Richmond [3] give the following analog of Theorem 1.1.

Theorem 1.6. If $\mathcal{K}$ is a multiplicatively adequate class of finite structures and

$$
F_{\mathcal{K}}(x)=\sum_{1 \leq n \leq x} f_{\mathcal{K}}(n)
$$

is slowly varying at infinity, then the class $\mathcal{K}$ has a first order 0-1 law, that is, given any sentence in first order logic, the probability that it holds in $\mathcal{K}$ is always either 0 or 18

We prove the following analog of Theorem 1.3 (this theorem belongs to multiplicative number theory).

Theorem 1.7. Suppose $f$ and $g$ are functions with nonnegative integer values satisfying Identity 圆, If

$$
G(x)=\sum_{2 \leq n \leq x} g(n)=\mathrm{O}\left((\log x)^{k}\right)
$$

for some $k$, then $F(x)=\sum_{1 \leq n \leq x} f(n)$ is slowly varying at infinity.

Combining Theorems 1.6 and 1.7 we obtain the following theorem.

Theorem 1.8. If $\mathcal{K}$ is a multiplicatively adequate class of finite structures such that

$$
G_{\mathcal{K}}(x)=\sum_{2 \leq n \leq x} g_{\mathcal{K}}(n)=\mathrm{O}\left((\log x)^{k}\right)
$$

for some $k$, then $\mathcal{K}$ has a first order $0-1$ law.

This theorem covers the key result Theorem 3.5 of Burris, Compton, Odlyzko and Richmond [3]. An infinite subclass $\mathcal{Q}$ of $\mathcal{K}$ is approximately exponential if the sequence of logarithms of the sizes of members of $\mathcal{Q}$ is asymptotic to a linear function of $n$. They prove that if $\mathcal{K}$ is a multiplicatively adequate class of finite structures such that the collection of indecomposables can be expressed as a finite

\footnotetext{
${ }^{7}$ The fact that the size of the direct product of two structures is the product of their sizes leads to the classification of counting problems for such classes as belonging to the multiplicative case.

${ }^{8}$ In the multiplicative case, the probability that a sentence holds is defined to be the limit as $n \rightarrow \infty$ of the proportion of structures of size less than or equal to $n$ in $\mathcal{K}$ that satisfy the sentence, provided such limit exists.
} 
union of classes, each of which is either finite or approximately exponential, then $\mathcal{K}$ has a first-order $0-1$ law.

Theorem 1.8 also covers one of the main results, Theorem 5.28, of Burris and Sárközy [4]. A multiplicatively adequate class $\mathcal{K}$ is said to be discrete if there is an integer $d$ such that all members of $\mathcal{K}$ have sizes that are powers of $d$. For such $\mathcal{K}$ they prove that if $g_{\mathcal{K}}(n)$ is uniformly bounded then $\mathcal{K}$ has a first-order 0-1 law. 9

\section{Proofs of AdDitive Results}

In this section we adopt the convention of 2 that upper case bold letters name power series whose coefficients are given by the corresponding lower case italic letters, for example

$$
\mathbf{F}(x)=\sum_{n=0}^{\infty} f(n) x^{n} .
$$

By this convention $\mathbf{A}(x)$ is the power series $\sum a(n) x^{n}$ and $\mathbf{A}_{1}(x)$ is the power series $\sum a_{1}(n) x^{n}$, etc.

Lemma 2.1. Suppose $\mathbf{F}(x)$ is a power series with nonnegative coefficients and suppose that $f(n-j) / f(n)=1+\mathrm{o}(1)$ for all $j$ in some nonempty finite set $S$ of positive integers. Then $f(n-d) / f(n)=1+\mathrm{o}(1)$, where $d=\operatorname{gcd}(S)$.

Proof. Notice that if $m$ is any positive integer, and $j \in S$, then

$$
\begin{aligned}
f(n-m j) / f(n) & =\prod_{\ell=1}^{m} f(n-\ell j) / f(n-(\ell-1) j) \\
& =\prod_{\ell=1}^{m}(1+\mathrm{o}(1)) \\
& =1+\mathrm{o}(1) .
\end{aligned}
$$

We also have $f(n+j) / f(n)=1+o(1)$, so in the same manner $f(n+m j) / f(n)=$ $1+\mathrm{o}(1)$ for any positive integer $m$. Combining these facts, we see that if $j \in S$ then

$$
f(n+m j) / f(n)=1+o(1)
$$

for any fixed integer $m$. Let $\left\{j_{1}, \ldots, j_{r}\right\}$ be a listing of all elements of $S$. Choose integers $m_{1}, \ldots, m_{r}$ such that $m_{1} j_{1}+\cdots+m_{r} j_{r}=d$. Then

$$
\begin{aligned}
& f(n-d) / f(n) \\
= & f\left(n-m_{1} j_{1}-\cdots-m_{r} j_{r}\right) / f(n) \\
= & \prod_{k=1}^{r} f\left(\left(n-m_{1} j_{1}-\cdots-m_{k-1} j_{k-1}\right)-m_{k} j_{k}\right) / f\left(n-m_{1} j_{1}-\cdots-m_{k-1} j_{k-1}\right) \\
= & \left.\prod_{k=1}^{r}(1+\mathrm{o}(1)) \quad \text { (by equation (2.1) }\right) \\
= & 1+\mathrm{o}(1) .
\end{aligned}
$$

This completes the proof of the lemma.

\footnotetext{
${ }^{9}$ For multiplicatively adequate classes $\mathcal{K}$ that are discrete there is a version of Theorem 1.8 that uses the probability defined by local counting (instead of global counting).
} 
Lemma 2.2. Suppose that $f$ and a are functions with nonnegative integer values and suppose there are positive integers

$$
j_{1}, j_{2}, \ldots, j_{m}
$$

such that

$$
\mathbf{A}(x)=\left(\prod_{k=1}^{m}\left(1-x^{j_{k}}\right)\right) \mathbf{F}(x) .
$$

If $a(n)=\mathrm{o}(f(n))$ and $m>1$, then

$$
a_{1}(n)=\mathrm{o}(f(n))
$$

where

$$
\mathbf{A}_{1}(x)=\left(1-x^{j_{m}}\right)^{-1} \mathbf{A}(x) .
$$

Proof. Note that $f(n)$ is eventually positive as $a(n)=o(f(n))$. Suppose that $a_{1}(n) \neq \mathrm{o}(f(n))$. Then there exists a constant $C>0$ such that

$$
\limsup _{n \rightarrow \infty} a_{1}(n) / f(n)>C .
$$

Let $R$ be an integer such that

$$
R>3 j_{1} / C
$$

From $a(n)=\mathrm{o}(f(n))$ and item (2.2) we can choose $N>R j_{1} j_{m}$ such that

$$
\begin{aligned}
a_{1}(N) & >C f(N) \quad \text { and } \\
a(n) & <R^{-2} f(n) \quad \text { for all } n>N-R j_{1} j_{m} .
\end{aligned}
$$

From item (2.5) one has

$$
a\left(N-\ell j_{m}\right)<R^{-2} f\left(N-\ell j_{m}\right) \quad \text { for } \ell<R j_{1} .
$$

Notice, for $q \leq R j_{1}$, that

$$
\begin{aligned}
a_{1}\left(N-q j_{m}\right) & =a_{1}(N)-\sum_{\ell=0}^{q-1}\left(a_{1}\left(N-\ell j_{m}\right)-a_{1}\left(N-(\ell+1) j_{m}\right)\right) \\
& =a_{1}(N)-\sum_{\ell=0}^{q-1} a\left(N-\ell j_{m}\right) \\
& \left.>C f(N)-\sum_{\ell=0}^{q-1} R^{-2} f\left(N-\ell j_{m}\right) \quad \text { (by items (2.4) and (2.6) }\right) \\
& \geq C f(N)-q R^{-2} f(N),
\end{aligned}
$$

where the last line follows from the fact that $f(N) \geq f\left(N-\ell j_{m}\right)$ for $0 \leq \ell \leq R j_{1}$. Letting $q=r j_{1}$ gives

$$
a_{1}\left(N-r j_{1} j_{m}\right)>\left(C-r j_{1} R^{-2}\right) f(N) \quad \text { for } 0 \leq r \leq R .
$$

Notice that

$$
\mathbf{F}(x)=\left(\prod_{k=1}^{m-1}\left(1-x^{j_{k}}\right)^{-1}\right) \mathbf{A}_{1}(x) .
$$


We also have for $r \geq 0$

$$
\left[x^{r j_{1} j_{m}}\right] \prod_{k=1}^{m-1}\left(1-x^{j_{k}}\right)^{-1} \geq\left[x^{r j_{1} j_{m}}\right]\left(1-x^{j_{1}}\right)^{-1}=1 .
$$

Thus

$$
\begin{aligned}
f(N) & =\sum_{j=0}^{N}\left(\left[x^{j}\right] \prod_{k=1}^{m-1}\left(1-x^{j_{k}}\right)^{-1}\right) a_{1}(N-j) \\
& \geq \sum_{r=0}^{R-1}\left(\left[x^{r j_{1} j_{m}}\right] \prod_{k=1}^{m-1}\left(1-x^{j_{k}}\right)^{-1}\right) a_{1}\left(N-r j_{1} j_{m}\right) \\
& >\sum_{r=0}^{R-1}\left(C-r j_{1} R^{-2}\right) f(N) \quad(\text { by items (2.7) and (2.8) }) \\
& =\left(R C-R(R-1) j_{1} R^{-2} / 2\right) f(N) \\
& \geq \frac{5 j_{1} f(N)}{2} \quad(\text { by item } \underline{(2.3)}) \\
& \geq \frac{5}{2} f(N) .
\end{aligned}
$$

This is a contradiction as $f(N)>0$. Hence $a_{1}(n)=\mathrm{o}(f(n))$ as required.

Corollary 2.3. Let $\mathbf{F}(x)$ and $\mathbf{A}(x)$ be as in the preceding lemma with $m \geq 1$, and suppose that $a(n)=\mathrm{o}(f(n))$. Then

$$
f\left(n-j_{1}\right) / f(n) \rightarrow 1 \quad \text { as } n \rightarrow \infty .
$$

Proof. The claim is proved by induction on $m$. When $m=1$ we have $a(n)=$ $f(n)-f\left(n-j_{1}\right)$, so $f(n)-f\left(n-j_{1}\right)=\mathrm{o}(f(n))$. Hence $f\left(n-j_{1}\right) / f(n)=1+\mathrm{o}(1)$, so the claim is true when $m=1$. Suppose the claim is true for $m<\ell \geq 2$, and consider the case that $m=\ell$. By the preceding lemma

$$
\mathbf{A}_{1}(x)=\left(1-x^{j_{\ell}}\right)^{-1} \mathbf{A}(x)
$$

satisfies $a_{1}(n)=\mathrm{o}(f(n))$. Notice also that

$$
\mathbf{F}(x)=\left(\prod_{k=1}^{\ell-1}\left(1-x^{j_{k}}\right)^{-1}\right) \mathbf{A}_{1}(x)
$$

and hence by the inductive hypothesis $f\left(n-j_{1}\right) / f(n)=1+\mathrm{o}(1)$. Thus the claim is true for all $m$.

We are now ready to prove our main additive result, Theorem 1.3

Theorem 2.4. Suppose $f$ and $g$ are functions with nonnegative integer values satisfying Identity [1, In addition, suppose that g satisfies:

(a) $\operatorname{gcd}(\{n: g(n) \neq 0\})=1$;

(b) $g(n) \leq C n^{k}$ for some $k$ and some $C>0$.

Then

$$
f(n-1) / f(n) \rightarrow 1 \quad \text { as } n \rightarrow \infty
$$


Proof. If $\{n: g(n) \neq 0\}$ is finite, then the conclusion follows from a theorem of Schur (see Wilf [10], Theorem 3.15.2), which says that $f(n)$ is asymptotic to a polynomial in $n$. Now consider the case that $\{n: g(n) \neq 0\}$ is infinite. Choose $N$ such that

$$
\begin{aligned}
& r:=\sum_{n=1}^{N} g(n) \geq k+4 \quad \text { and } \\
& \operatorname{gcd}(\{n \leq N: g(n) \neq 0\})=1 .
\end{aligned}
$$

By Schur's Theorem [10] there is a positive constant $C_{0}$ such that

$$
\left[x^{n}\right] \prod_{j=1}^{N}\left(1-x^{j}\right)^{-g(j)} \sim C_{0} n^{r-1},
$$

so we have

$$
\left[x^{n}\right] \prod_{j=1}^{N}\left(1-x^{j}\right)^{-g(j)}>C n^{k+2}
$$

for all $n$ greater than or equal to some $N_{1}$. Take $M=\max \left(N, N_{1}\right)$. Let

$$
\mathbf{A}(x)=\left(\prod_{n=1}^{M}\left(1-x^{n}\right)^{g(n)}\right) \mathbf{F}(x) .
$$

We claim that $a(n)=\mathrm{o}(f(n))$. To see this, notice by Identity 1 that

$$
\mathbf{A}(x)=\exp (\mathbf{H}(x))
$$

where

$$
\mathbf{H}(x)=\sum_{n=M+1}^{\infty} g(n) x^{n}+\frac{1}{2} \sum_{n=M+1}^{\infty} g(n) x^{2 n}+\frac{1}{3} \sum_{n=M+1}^{\infty} g(n) x^{3 n}+\cdots .
$$

Thus

$$
\begin{aligned}
& h(n)=0 \quad \text { if } n \leq M, \text { and } \\
& h(n) \leq \frac{1}{n} \sum_{d \mid n} d g(d)<C n^{k+1} \quad \text { if } n>M .
\end{aligned}
$$

Differentiating equation (2.12) gives $\mathbf{A}^{\prime}(x)=\mathbf{A}(x) \mathbf{H}^{\prime}(x)$, and then equating the coefficient of $x^{n-1}$ from both sides leads to the following for $n>M+1$ :

$$
\begin{aligned}
n a(n) & =\sum_{i=M+1}^{n-1} a(n-i) i h(i) \quad \text { by item (2.13) } \\
& \leq \sum_{i=M+1}^{n} a(n-i) C i^{k+2} \quad \text { by item (2.14) } \\
& \left.\leq \sum_{i=M+1}^{n} a(n-i)\left(\left[x^{i}\right] \prod_{j=1}^{M}\left(1-x^{j}\right)^{-g(j)}\right) \quad \text { by item (2.11) }\right) \\
& \leq \sum_{i=0}^{n} a(n-i)\left(\left[x^{i}\right] \prod_{j=1}^{M}\left(1-x^{j}\right)^{-g(j)}\right) \\
& =f(n) .
\end{aligned}
$$


Thus $f(n) \geq n a(n)$ for $n>M+1$, so $a(n)=o(f(n))$. From Corollary 2.3, it follows that for any $j \leq M$ such that $g(j) \neq 0$, we have $f(n-j) / f(n)=1+\mathrm{o}(1)$. Since $\operatorname{gcd}(\{j \leq M: g(j) \neq 0\})=1$, by Lemma 2.1 we have $f(n-1) / f(n) \rightarrow 1$ as $n \rightarrow \infty$. This completes the proof of the theorem.

Now we can give the

Proof of Theorem 1.5. This follows immediately from Theorems [1.1] and 2.4]

It should be noted that if either condition $(a)$ or $(b)$ given in Theorem 2.4 is relaxed, then Theorem 2.4 will not be true. This fact was known to Burris and Sárközy (see page 492 of [4]) who had constructions showing that the conclusion of Theorem 2.4 would not necessarily hold if condition (b) failed to hold; since these constructions were omitted from their paper we will prove the following proposition.

Proposition 2.5. Suppose either condition (a) does not hold, or condition (b) is replaced by $g(n)=\mathrm{O}(t(n))$, where $t(n)$ is some function satisfying

$$
\limsup _{n \rightarrow \infty} \log t(n) / \log n=\infty .
$$

Then the conclusion of Theorem 2.4 does not necessarily hold. In fact, if condition (b) fails, the function $g$ can be chosen such that

$$
\sum_{n=0}^{\infty} f(n) x^{n}=\prod_{n=1}^{\infty}\left(1-x^{n}\right)^{-g(n)}
$$

satisfies $\liminf _{n \rightarrow \infty}(f(1)+f(2)+\cdots+f(n-1)) / f(n)=0$.

Proof. Suppose condition $(a)$ fails. Then $d>1$ for $d=\operatorname{gcd}(\{n: g(n) \neq 0\})$. Since $f(n)=0$ whenever $n$ is not a multiple of $d$, the conclusion of Theorem 2.4 does not hold. Next suppose we replace condition $(b)$ of Theorem 2.4 by $g(n)=\mathrm{O}(t(n))$, where $t(n)$ is some function that satisfies

$$
\limsup _{n \rightarrow \infty} \log t(n) / \log (n)=\infty \text {. }
$$

Let $g(1)=1$, and define a set $\mathcal{N}=\left\{n_{1}, n_{2}, \ldots\right\}$ of positive integers as follows. Take $n_{1}=2$, and if $n_{1}, \ldots, n_{k}$ are defined, note that

$$
\lim _{n \rightarrow \infty} \log \left(\begin{array}{c}
n-1+\sum_{i=1}^{k}\left\lfloor t\left(n_{i}\right)\right\rfloor \\
\sum_{i=1}^{k}\left\lfloor t\left(n_{i}\right)\right\rfloor
\end{array}\right) / \log n=\sum_{i=1}^{k}\left\lfloor t\left(n_{i}\right)\right\rfloor .
$$

Choose $n_{k+1}$ such that

$$
t\left(n_{k+1}\right)>n_{k+1}^{2}\left(\begin{array}{c}
n_{k+1}-1+\sum_{i=1}^{k}\left\lfloor t\left(n_{i}\right)\right\rfloor \\
\sum_{i=1}^{k}\left\lfloor t\left(n_{i}\right)\right\rfloor
\end{array}\right) .
$$

This is possible by equations (2.15) and (2.16). Now for $n \geq 2$ define

$$
g(n)= \begin{cases}\lfloor t(n)\rfloor & \text { if } n \in \mathcal{N} \\ 0 & \text { otherwise }\end{cases}
$$

Notice that $g(n)=\mathrm{O}(t(n))$; moreover, $f\left(n_{k}\right) \geq g\left(n_{k}\right)$, whereas for $m<n_{k}$, 


$$
\begin{aligned}
f(m) & =\left[x^{m}\right] \prod_{j=1}^{n_{k}-1}\left(1-x^{j}\right)^{-g(j)} \\
& =\left[x^{m}\right](1-x)^{-1} \prod_{j=1}^{k-1}\left(1-x^{n_{j}}\right)^{-\left\lfloor t\left(n_{j}\right)\right\rfloor} \\
& \leq\left[x^{m}\right](1-x)^{-1-\sum_{1 \leq j<k}\left\lfloor t\left(n_{j}\right)\right\rfloor} \\
& =\left(\begin{array}{c}
m+\sum_{1 \leq j<k}\left\lfloor t\left(n_{j}\right)\right\rfloor \\
\sum_{1 \leq j<k}\left\lfloor t\left(n_{j}\right)\right\rfloor
\end{array}\right) \\
& \leq\left(\begin{array}{c}
n_{k}-1+\sum_{1 \leq j<k}\left\lfloor t\left(n_{j}\right)\right\rfloor \\
\sum_{1 \leq j<k}\left\lfloor t\left(n_{j}\right)\right\rfloor
\end{array}\right) \\
& \leq t\left(n_{k}\right) / n_{k}^{2} \quad(\text { by item }(2.17)) \\
& \leq\left(g\left(n_{k}\right)+1\right) / n_{k}^{2} \\
& \leq\left(f\left(n_{k}\right)+1\right) / n_{k}^{2} .
\end{aligned}
$$

Hence

$$
f(1)+\cdots+f\left(n_{k}-1\right) \leq\left(f\left(n_{k}\right)+1\right) / n_{k}=\mathrm{o}\left(f\left(n_{k}\right)\right)
$$

as $k \rightarrow \infty$. In particular, $f\left(n_{k}-1\right) / f\left(n_{k}\right)=\mathrm{o}(1)$, and so $f(n-1) / f(n) \nrightarrow 1$.

\section{Proofs of multiplicative Results}

In this section we adopt the convention of 22 that upper case bold letters name Dirichlet series whose coefficients are given by the corresponding lower case italic letters, for example

$$
\mathbf{F}(s)=\sum_{n=1}^{\infty} f(n) n^{-s} .
$$

By this convention $\mathbf{A}(s)$ is the Dirichlet series $\sum a(n) n^{-s}$ and $\mathbf{A}_{1}(s)$ is the Dirichlet series $\sum a_{1}(n) n^{-s}$, etc.

The next definition explains that the corresponding capital italic letters are used for global counts of Dirichlet series.

Definition 3.1. Given a Dirichlet series $\mathbf{F}(s)$, we say that

$$
F(x)=\sum_{1 \leq n \leq x} f(n)
$$

is the global counting function of $\mathbf{F}(s)$. Furthermore, let

$$
\bar{F}(x)=\sum_{1 \leq n \leq x} f(n) \log n,
$$

be the global counting function of $-\mathbf{F}^{\prime}(s)$, the derivative of $-\mathbf{F}(s)$ with respect to $s$.

The global counting function $F(x)$ of a Dirichlet series $\mathbf{F}(s)$ plays the role that the coefficients of a power series play in the additive case when considering the definition of the probability of a property holding in a class of structures.

The following simple lemma gives the asymptotic relationship between $F(x)$ and $\bar{F}(x)$ that we will need. 
Lemma 3.2. Suppose $\mathbf{F}(s)$ is a Dirichlet series with nonnegative coefficients such that $F(x) \rightarrow \infty$ as $x \rightarrow \infty$. Then $F(x)=\mathrm{o}(\bar{F}(x))$.

Proof. Let $\varepsilon>0$ and choose $N>1 / \varepsilon$ such that $F(x)>0$ for $x>e^{N}+1$. Notice that for $x>e^{N}+1$

$$
\begin{aligned}
\bar{F}(x) & =\sum_{1 \leq n \leq x} f(n) \log n \\
& \geq \sum_{e^{N}<n \leq x} f(n) \log n \\
& \geq N \sum_{e^{N}<n \leq x} f(n) \\
& =N F(x)-N F\left(e^{N}\right) .
\end{aligned}
$$

Hence for $x>e^{N}+1$

$$
\bar{F}(x) / F(x) \geq N-N F\left(e^{N}\right) / F(x) \rightarrow N \quad \text { as } x \rightarrow \infty .
$$

Thus

$$
\limsup _{x \rightarrow \infty} F(x) / \bar{F}(x) \leq \varepsilon
$$

and the lemma follows.

Definition 3.3. A function $T:[a, \infty) \rightarrow[0, \infty)$ which is eventually positive is in $R V_{0}$ if for any $\beta$ with $0<\beta<1$,

$$
T(\beta x) / T(x) \rightarrow 1
$$

as $x \rightarrow \infty$. (This is read as " $T$ has regular variation at infinity of index 0 ", or more popularly one says, " $T$ is slowly varying at infinity".)

Remark 3.4. If $T(x)$ is a nondecreasing function that is eventually positive, and for some positive number $\beta \neq 1$ we have

$$
T(\beta x) / T(x) \rightarrow 1
$$

as $x \rightarrow \infty$, then $T(x) \in R V_{0}$ (see [4, Corollary 4.3).

The theorems from the additive case lift nicely to the multiplicative case. First we rephrase Theorem 8.30 of [2] to fit the notation of this paper-this provides the multiplicative counterpart of Schur's Theorem. The central part of the proof given in [2] is an intuitive geometric proof, so we take this opportunity to present a rigorous proof.

Theorem 3.5. If $f$ and $g$ are functions with nonnegative integer values such that

$$
\mathbf{F}(s)=\prod_{n=2}^{M}\left(1-n^{-s}\right)^{-g(n)},
$$

then

$$
F(x)=(\log x)^{r} /\left(r !(\log 2)^{g(2)} \cdots(\log M)^{g(M)}\right)+\mathrm{O}\left((\log x)^{r-1}\right),
$$

where $r=\sum_{n=2}^{M} g(n)>0$.

Proof. We prove this by induction on $r$. Suppose $r=1$. Then 


$$
\sum_{n=1}^{\infty} f(n) n^{-s}=\left(1-k^{-s}\right)^{-1}
$$

for some $k$ with $2 \leq k \leq M$. Hence $f(n)$ is equal to one if $n$ is a power of $k$ and is zero otherwise, so

$$
F(x)=\lfloor\log x / \log k\rfloor=(\log x) /(1 !(\log k))+\mathrm{O}(1),
$$

so the claim is true for $r=1$. Now suppose the claim is true for all $r<m \geq 2$ and consider the case when $r=m$. Choose $j$ with $2 \leq j \leq M$ such that $g(j)>0$ and let

$$
\begin{aligned}
\mathbf{F}_{1}(s) & =\left(1-j^{-s}\right) \mathbf{F}(s), \\
L & =m !(\log 2)^{g(2)} \cdots(\log M)^{g(M)} .
\end{aligned}
$$

By the inductive hypothesis there exists a constant $C>0$ such that

$$
\left|F_{1}(x)-(\log x)^{m-1} m(\log j) / L\right|<C\left((\log x)^{m-2}+1\right)
$$

for all $x \geq 1$. From

$$
F(x)=\sum_{n=0}^{\lfloor\log x / \log j\rfloor} F_{1}\left(x / j^{n}\right)
$$

it follows that

$$
\begin{aligned}
& \left|F(x)-\sum_{n=0}^{\lfloor\log x / \log j\rfloor}\left(\log \left(x / j^{n}\right)\right)^{m-1} m(\log j) / L\right| \\
= & \left|\sum_{n=0}^{\lfloor\log x / \log j\rfloor} F_{1}\left(x / j^{n}\right)-\sum_{n=0}^{\lfloor\log x / \log j\rfloor}\left(\log \left(x / j^{n}\right)\right)^{m-1} m(\log j) / L\right| \\
\leq & \sum_{n=0}^{\lfloor\log x / \log j\rfloor} C\left((\log x)^{m-2}+1\right) \quad(\text { by item (B.1) }) \\
= & (1+\lfloor\log x / \log j\rfloor) \cdot C\left((\log x)^{m-2}+1\right) \\
= & \mathrm{O}\left((\log x)^{m-1}\right) \quad(\text { since } m \geq 2) .
\end{aligned}
$$

Hence

$$
F(x)=\sum_{n=0}^{\lfloor\log x / \log j\rfloor}\left(\log \left(x / j^{n}\right)\right) m(\log j) / L+\mathrm{O}\left((\log x)^{m-1}\right) .
$$

We also have

$$
\begin{aligned}
& \sum_{n=0}^{\lfloor\log x / \log j\rfloor}\left(\log \left(x / j^{n}\right)\right)^{m-1} m(\log j) / L \\
= & m(\log j) / L \sum_{n=0}^{\lfloor\log x / \log j\rfloor}(\log x-n \log j)^{m-1} \\
= & m(\log j)^{m} / L \sum_{n=0}^{\lfloor\log x / \log j\rfloor}((\log x / \log j)-n)^{m-1} .
\end{aligned}
$$


It is a well-known fact that

$$
\sum_{0 \leq n \leq u}(u-n)^{m-1}=\int_{0}^{u} t^{m-1} d t+\mathrm{O}\left(u^{m-1}\right)=u^{m} / m+\mathrm{O}\left(u^{m-1}\right) .
$$

Hence

$$
\begin{aligned}
& m(\log j)^{m} / L \sum_{n=0}^{\lfloor\log x / \log j\rfloor}((\log x / \log j)-n)^{m-1} \\
= & (\log x)^{m} / L+\mathrm{O}\left((\log x)^{m-1}\right) .
\end{aligned}
$$

Using this fact and equations (3.2) and (3.3) we deduce that

$$
F(x)=(\log x)^{m} / L+\mathrm{O}\left((\log x)^{m-1}\right),
$$

completing the induction proof.

Lemma 3.6. Suppose that $f$ and $a$ are functions with nonnegative integer values and suppose there are positive integers

$$
2 \leq j_{1} \leq j_{2} \leq \cdots \leq j_{m}
$$

such that

$$
\mathbf{A}(s)=\left(\prod_{i=1}^{m}\left(1-j_{i}^{-s}\right)\right) \mathbf{F}(s)
$$

If $A(x)=\mathrm{o}(F(x))$ and $m>1$, then

$$
A_{1}(x)=\mathrm{o}(F(x))
$$

where

$$
\mathbf{A}_{1}(s)=\left(1-j_{m}^{-s}\right)^{-1} \mathbf{A}(s)
$$

Proof. Suppose that $A_{1}(x) \neq \mathrm{o}(F(x))$. Then there exists a constant $C>0$ such that

$$
\limsup _{x \rightarrow \infty} A_{1}(x) / F(x)>C .
$$

Let $R$ be an integer satisfying

$$
R>3 / C
$$

and choose $\varepsilon>0$ such that

$$
R(R+1) \varepsilon / 2<1 .
$$

From $A(x)=\mathrm{o}(F(x))$ and item (3.4) we can choose $y$ such that

$$
\begin{aligned}
A(y) & <\varepsilon F(y), \\
A_{1}(y) & >C F(y) .
\end{aligned}
$$

We have for $r \geq 1$

$$
\begin{aligned}
A_{1}\left(y / j_{m}^{r}\right) & =A_{1}(y)-\sum_{i=1}^{r}\left(A_{1}\left(y / j_{m}^{i-1}\right)-A_{1}\left(y / j_{m}^{i}\right)\right) \\
& =A_{1}(y)-\sum_{i=1}^{r} A\left(y / j_{m}^{i-1}\right) \\
& \geq A_{1}(y)-r A(y) \\
& >C F(y)-r \varepsilon F(y) \quad \text { (by items (3.7) and (3.8) }),
\end{aligned}
$$


hence for $r \geq 1$

$$
A_{1}\left(y / j_{m}^{r}\right) \geq(C-r \varepsilon) F(y) .
$$

But,

$$
\begin{aligned}
F(y) & \geq \sum_{r=1}^{R} A_{1}\left(y / j_{1}^{r}\right) \\
& \geq \sum_{r=1}^{R} A_{1}\left(y / j_{m}^{r}\right) \quad\left(\text { since } j_{m} \geq j_{1}\right) \\
& \geq \sum_{r=1}^{R}(C-r \varepsilon) F(y) \quad(\text { by item (3.9) }) \\
& =(C R-R(R+1) \varepsilon / 2) F(y) \\
& \geq 2 F(y) \quad(\text { by items (3.5) and (‥6) }) .
\end{aligned}
$$

This is a contradiction as $F(y)>0$. The claim follows.

From this lemma we obtain a useful corollary.

Corollary 3.7. Let $\mathbf{A}(s)$ and $\mathbf{F}(s)$ be as in the preceding lemma with $m \geq 1$. If $A(x)=\mathrm{o}(F(x))$, then $F(x) \in R V_{0}$.

Proof. The proof is by induction on $m$. For the case $m=1$ we have $A(x)=$ $F(x)-F\left(x / j_{1}\right)$, so $F(x)-F\left(x / j_{1}\right)=\mathrm{o}(F(x))$. Hence $F\left(x / j_{1}\right) / F(x) \rightarrow 1$, and so $F(x) \in R V_{0}$ by Remark 3.4 Suppose the claim is true for $m<\ell \geq 2$, and consider the case when $m=\ell$. Let

$$
\mathbf{A}_{1}(s)=\left(1-j_{\ell}^{-s}\right)^{-1} \mathbf{A}(s) .
$$

The preceding lemma shows that $A_{1}(x)=\mathrm{o}(F(x))$. Also we have

$$
\mathbf{F}(s)=\left(\prod_{k=1}^{\ell-1}\left(1-j_{k}^{-s}\right)^{-1}\right) \mathbf{A}_{1}(s)
$$

and hence by the inductive hypothesis $F(x) \in R V_{0}$. Thus the claim is true for all $m$.

We are now ready to prove our main multiplicative result, Theorem 1.7 .

Theorem 3.8. Let $f$ and $g$ be functions with nonnegative integer values satisfying Identity 园, If

$$
G(x) \leq C(\log x)^{k}
$$

for some constants $C$ and $k$, then $F(x) \in R V_{0}$.

Proof. If $\{n: g(n) \neq 0\}$ is finite then by Theorem 3.5 there exists a positive constant $C_{0}$ such that $F(x) \sim C_{0}(\log x)^{r}$, where $r=\sum_{n \geq 2} g(n)$, and thus $F(x) \in R V_{0}$. Hence we may assume that $\{n: g(n) \neq 0\}$ is infinite. Choose $N$ such that

$$
\sum_{n=2}^{N} g(n) \geq k+2 \text {. }
$$


Let

$$
\mathbf{A}(s)=\prod_{n=N+1}^{\infty}\left(1-n^{-s}\right)^{-g(n)} .
$$

We shall show that $A(x)=\mathrm{o}(F(x))$. Let

$$
h(n)=\sum_{\substack{j^{\ell}=n \\ j>N}} g(j) / \ell \quad \text { for } n>N .
$$

Notice that $\mathbf{H}(s)=\sum_{n=N+1}^{\infty} h(n) n^{-s}$ satisfies $\mathbf{A}(s)=\exp (\mathbf{H}(s))$ (see Identity 2). Differentiating this equation gives

$$
\mathbf{A}^{\prime}(s)=\mathbf{A}(s) \mathbf{H}^{\prime}(s) .
$$

Notice that for $x>N+1$

$$
\begin{aligned}
\bar{H}(x) & =\sum_{\substack{N<n \leq x\\
}} h(n) \log n \\
& \sum_{\substack{j^{\ell} \leq x \\
N<j}}(g(j) / \ell) \log \left(j^{\ell}\right) \\
& \leq \sum_{\left.\substack{N<j \leq x \\
\leq} \frac{\log x}{\log j}\right\rfloor g(j) \log j} \\
& \leq C(\log x) G(x) \\
&
\end{aligned}
$$

Using the factorization of $\mathbf{A}^{\prime}(s)$ in equation (3.11) gives

$$
\bar{A}(x)=\sum_{1 \leq n \leq x} a(n) \bar{H}(x / n)
$$

(see Theorem 3.10 of [1]). Let

$$
\mathbf{F}_{1}(s)=\prod_{n=2}^{N}\left(1-n^{-s}\right)^{-g(n)} .
$$

Using Theorem 3.5 and item (3.10), it is possible to choose $M>N+1$ such that $F_{1}(x) \geq C(\log x)^{k+1}$ for all $x \geq M$. Then by item (3.12)

$$
F_{1}(x) \geq \bar{H}(x) \quad \text { for } x \geq M .
$$

We have

$$
\mathbf{F}(s)=\mathbf{A}(s) \mathbf{F}_{1}(s),
$$

and hence for $x \geq M$

$$
\begin{aligned}
F(x) & =\sum_{1 \leq n \leq x} a(n) F_{1}(x / n) \\
& \geq \sum_{1 \leq n \leq x / M} a(n) F_{1}(x / n) \\
& \geq \sum_{1 \leq n \leq x / M} a(n) \bar{H}(x / n) \quad \text { by item }
\end{aligned}
$$




$$
\begin{aligned}
& =\sum_{1 \leq n \leq x} a(n) \bar{H}(x / n)-\sum_{x / M<n \leq x} a(n) \bar{H}(x / n) \\
& =\bar{A}(x)-\sum_{x / M<n \leq x} a(n) \bar{H}(x / n) \\
& \geq \bar{A}(x)-\bar{H}(M) A(x) \\
& =\bar{A}(x)+\mathrm{O}(A(x)) .
\end{aligned}
$$

By Lemma 3.2, we know that $A(x)=\mathrm{o}(\bar{A}(x))$ and hence $A(x)=\mathrm{o}(F(x))$. Then Corollary 3.7 shows that $F(x) \in R V_{0}$. This completes the proof.

And now we can give the

Proof of Theorem 1.8, This follows immediately from Theorems 1.6 and 3.8

The condition

$$
G(x)=\mathrm{O}\left((\log x)^{k}\right)
$$

stated in the preceding theorem is, in a natural sense, the weakest hypothesis possible.

Proposition 3.9. Let $f$ and $g$ be as in the preceding theorem. If $G(x)=\mathrm{O}(T(x))$, where $T(x)$ is a nondecreasing function satisfying

$$
\limsup _{n \rightarrow \infty} \log T(n) / \log \log n=\infty,
$$

then the conclusion of Theorem 3.8 will not necessarily hold.

Proof. Notice that if we require that $g(n)=0$ whenever $n$ is not a power of 2 , then

$$
\mathbf{F}(s)=\prod_{n=2}^{\infty}\left(1-n^{-s}\right)^{-g(n)}=\prod_{n=1}^{\infty}\left(1-2^{-n s}\right)^{-g\left(2^{n}\right)} .
$$

Hence

$$
f\left(2^{n}\right)=\left[x^{n}\right] \prod_{n=1}^{\infty}\left(1-x^{n}\right)^{-g\left(2^{n}\right)} .
$$

Let $t(n)=T\left(2^{n}\right)-T\left(2^{n-1}\right)$ for all $n>1$ and let $t(1)=T(2)$. Since $T$ is nondecreasing, $t$ is a nonnegative valued function. We claim that

$$
\limsup _{n \rightarrow \infty} \log t(n) / \log n=\infty .
$$

To see this, notice that if there exist positive numbers $C$ and $k$ such that $t(n)<C n^{k}$ for all $n$, then

$$
T\left(2^{n}\right)=t(n)+t(n-1)+\cdots+t(1)<C\left(1+2^{k}+\cdots+n^{k}\right)<C n^{k+1} .
$$

Given $m$, choose $r$ such that $m \leq 2^{r}<2 m$. Hence

$$
T(m) \leq T\left(2^{r}\right)<C r^{k+1}<C\left(\log _{2} 2 m\right)^{k+1}=\mathrm{O}\left((\log m)^{k+1}\right) .
$$

This contradicts the fact that

$$
\limsup _{m \rightarrow \infty} \log T(m) /(\log \log m)=\infty .
$$


Hence equation (3.14) holds. It follows from Proposition 2.5 that we can choose values of $g(1), g(2), g(4), g(8), \ldots$ such that $g\left(2^{n}\right)=\mathrm{O}(t(n))$ and

$$
\liminf _{n \rightarrow \infty}\left(f\left(2^{n-1}\right)+f\left(2^{n-2}\right)+\cdots+f\left(2^{1}\right)\right) / f\left(2^{n}\right)=0 .
$$

Hence

$$
F(x) / F(2 x) \not \rightarrow 1
$$

as $x \rightarrow \infty$ and thus $F \notin R V_{0}$.

\section{Applications}

Example 4.1. Let $\mathcal{G}_{m, k}$ denote the collection of finite graphs that are forests in which each tree has at most $m$ vertices of total degree greater than 2 , and each vertex has total degree at most $k \cdot \mathcal{G}_{m, k}$ is additively adequate. The indecomposables of size $n$ consist of all trees with $n$ vertices that are elements of $\mathcal{G}_{m, k}$. Let $g_{m, k}(n)$ denote the number of indecomposables of size $n$. We show that $g_{m, k}(n)$ is polynomially bounded, arguing by induction on $m$. When $m=0$, the claim is easily seen to be true. Suppose the claim is true for all $m<r$. Notice that there is only one indecomposable of size $n$ with every vertex of degree at most two, namely the linear tree on $n$ vertices. This tree can be formed by taking a linear tree on $n-1$ vertices, creating a new vertex, and adjoining one of the end vertices of the linear tree on $n-1$ vertices to this new vertex. On the other hand, if $\mathcal{T}$ is an indecomposable of size $n$ having at least one vertex of total degree greater than 2 then removing some vertex of total degree at least three leaves us with $\ell$ trees for some $\ell$ between 3 and $k$. Each of the remaining trees can have at most $r-1$ vertices of degree greater than 2 , since $\mathcal{T}$ has at most $r$ vertices of degree greater than 2 and the vertex of $\mathcal{T}$ that was removed had degree greater than 2 . Hence any tree in $\mathcal{G}_{r, k}$ can be constructed by taking $k$ trees (some of which may be empty) from $\mathcal{G}_{r-1, k}$, choosing a vertex from each non-empty tree and creating a new tree by creating a new vertex, and connecting it to the chosen vertices from each of the trees. Of course, many trees other than those in $\mathcal{G}_{r, k}$ can be created by this procedure, but all that is important is that the trees in $\mathcal{G}_{r, k}$ can be obtained in this manner. Hence we obtain the estimate

$$
g_{r, k}(n) \leq n^{k}\left(1+g_{r-1, k}(1)+\cdots+g_{r-1, k}(n)\right)^{k},
$$

which is polynomially bounded. This completes the induction proof. It follows from Theorem 1.5 that $\mathcal{G}_{m, k}$ has a 0-1 law. In fact, we have shown that any subcollection of $\mathcal{G}_{m, k}$ that is additively adequate must have a monadic second order $0-1$ law, as it will also satisfy the polynomial bound condition of Theorem 1.5 It should also be noted that if $\mathcal{G}_{m, k}^{\prime}$ is the class of forests with trees satisfying the same properties as those in $\mathcal{G}_{m, k}$ with the additional property that the trees in the forest are rooted, then $\mathcal{G}_{m, k}^{\prime}$ has a 0-1 law. The reason for this is that given a tree of size $n$, selecting a rooting of this tree can create at most $n$ different trees. Hence if $g_{m, k}^{\prime}(n)$ denotes the number of indecomposables of size $n$ in $\mathcal{G}_{m, k}^{\prime}$, then $g_{m, k}^{\prime}(n)$ is at most $n$ times $g_{m, k}(n)$, so $g_{m, k}^{\prime}(n)$ is polynomially bounded as a function of $n$. Hence $\mathcal{G}_{m, k}^{\prime}$ has a monadic second order 0-1 law. Also, any subcollection of $\mathcal{G}_{m, k}^{\prime}$ that is additively adequate must have a monadic second order 0-1 law.

Example 4.2. Let $\mathcal{P}_{m, k}$ be the class of finite posets that are forests such that each tree in the forest has the property that each node has at most $k$ covers, and at most 
$m$ nodes have more than one cover. Then $\mathcal{P}_{m, k}$ is additively adequate. Just as in the preceding example, the number of indecomposables of $\mathcal{P}_{m, k}$ is polynomially bounded, so $\mathcal{P}_{m, k}$ has a monadic second order $0-1$ law by Theorem 1.5 .

Example 4.3. (Example 7.16 of Compton [5].) Let $\mathcal{E}$ denote the collection of finite equivalence relations. $\mathcal{E}$ is additively adequate. There is only one indecomposable of size $n$ for each $n \geq 1$. Hence the number of indecomposables is polynomially bounded, so $\mathcal{E}$ has a monadic second order $0-1$ law by Theorem[1.5. This fact also follows directly from Theorem 1.1 by using the formula for partitions of Rademacher [9], since the number of non-isomorphic equivalence relations of size $n$ is equal to $p(n)$, the number of partitions of $n$.

Example 4.4. (Example 8.3.4 of Burris [2].) Let $\mathcal{H}$ denote the collection of finite Heyting algebras in the equational class generated by the three element chain. $\mathcal{H}$ is multiplicatively adequate. There is one indecomposable of size $2^{n}+1$ for each $n$, and no other indecomposables. Hence the number of indecomposables of size less than $x$ is asymptotic to $\log _{2} x$. Thus by Theorem 1.8 this collection has a first order 0-1 law.

Example 4.5. Let $\mathcal{A}$ denote all finite $\mathbb{F}_{p}$-algebras with the property that their indecomposable factors are generated by a single element. This collection of algebras is multiplicatively adequate. The indecomposables consist of all algebras generated by a single element and having no non-trivial idempotents. Clearly, an indecomposable algebra of size $p^{n}$ is isomorphic to

$$
\mathbb{F}_{p}[x] /(q(x))
$$

for some polynomial of degree $n$. Factor $q(x)=\prod_{i=1}^{\ell} r_{i}(x)^{a_{i}}$, where $r_{1}, \ldots, r_{\ell}$ are distinct irreducible polynomials. By the Chinese remainder theorem,

$$
\mathbb{F}_{p}[x] /(q(x))=\prod_{i=1}^{\ell} \mathbb{F}_{p}[x] /\left(r_{i}(x)^{a_{i}}\right) .
$$

Hence if $\mathbb{F}_{p}[x] /(q(x))$ is indecomposable, $q(x)$ must be a power of an irreducible polynomial, say $q(x)=r(x)^{a}$. From [8]

$$
\mathbb{F}_{p}[x] /(q(x)) \cong \mathbb{F}_{p^{d}}[x] /\left(x^{a}\right),
$$

where $d$ is the degree of $r(x)$. Thus the number of indecomposable $\mathbb{F}_{p}$-algebras of size $p^{n}$ is equal to the number of algebras $\mathbb{F}_{p^{d}}[x] /\left(x^{a}\right)$ with $d a=n$, so the number is just $d(n)$, the number of divisors of $n$. Notice that

$$
G(x)=\sum_{p^{n} \leq x} d(n)=\sum_{n \leq \log x / \log p} d(n) .
$$

Since Theorem 320 in Hardy and Wright [7] says that

$$
\sum_{n \leq t} d(n) \sim t \log t
$$

as $t \rightarrow \infty$, it follows that $G(x)=\mathrm{O}(\log x \log \log x)=\mathrm{O}\left((\log x)^{2}\right)$. Thus, by Theorem 1.8, $\mathcal{A}$ has a first order 0-1 law.

Example 4.6. Let $\mathcal{G}$ be the collection of finite abelian $p$-groups for some prime $p$. Let $g(n)$ denote the number of indecomposables of size $n$. Then $g(n)=1$ if $n$ is a power of $p$ and zero otherwise. Hence $G(x)=\sum_{n \leq x} g(n)=\lfloor\log x / \log p\rfloor$. Thus $\mathcal{G}$ 
has a first order 0-1 law by Theorem 1.8. This can also be obtained from Theorem 1.1 by using Rademacher's formula for partitions [9], and the fact that the number of abelian $p$-groups of size $p^{n}$ is just $p(n)$, the number of partitions of $n$.

\section{REFERENCES}

[1] Tom M. Apostol, Introduction to Analytic Number Theory. Springer Verlag, 1976. MR 55:7892

[2] Stanley N. Burris, Number Theoretic Density and Logical Limit Laws. Mathematical Surveys and Monographs, 86. American Mathematical Society, Providence, RI, 2001. CMP 2001:05

[3] S. Burris, K. Compton, A. Odlyzko, and L.B. Richmond, Fine spectra and limit laws, II. First-order 0-1 laws. Can. J. Math. 49 (1997), 641-652. MR 98k:03076b

[4] S. Burris and A. Sárközy, Fine spectra and limit laws I. First-order laws. Can. J. Math. 49 (1997), 468-498. MR 98k:03076a

[5] K. Compton, A logical approach to asymptotic combinatorics. I. First order properties. Advances in Math. 65 (1987), 65-96. MR 88k:03065

[6] K. Compton, A logical approach to asymptotic combinatorics. II. Monadic second-order properties. Journal of Combinatorial Theory, Series A 50 (1989), 110-131. MR 90c:03024

[7] G. H. Hardy and E. M. Wright, An Introduction to the Theory of Numbers, Oxford Press, 5th ed., 1979. MR 81i:10002

[8] John Lawrence, personal communication.

[9] H. Rademacher, On the expansion of the partition function in a series. Ann. of Math. (2) 44 (1943), 416-422. MR 5:35a

[10] H. Wilf, Generatingfunctionology, Academic Press, 1990. MR 91g:05008

Department of Mathematics, University of California San Diego, La Jolla, CaliforNIA 92093-0112

E-mail address: jbell@math.ucsd.edu 